\title{
The significance of cardiac rhythm variability value assessment in partients wiht enfective endocarditis
}

\author{
Natalia Feaktsistava $^{1}$, Volga Sujayeva ${ }^{1}$, Swetlana Sudzhaeva ${ }^{1}$
}

1) Department of Rehabititation After Cardiac Surgery, Republican Scientific and Practical Centre of Cardiology, Minsk, Belarus

\section{Summary}

Objective: To assess heart rate variability (HRV) by the results of 24-hours ESG monitoring in patients with infection endocarditis (IE) before surgery and over a 6-month period after the surgical correction of valve heart pathology.

Material and Method: In 35 patients included into the study, an assessment of initial HRV (before surgery, I test) was performed

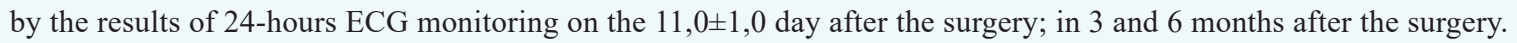

Results: More than a half of IE patients in pre-and early postoperative periods ( $56 \%$ and $57 \%$ respectively) were found to have hypersympaticotonia which was associated with the reduction of cardiac rhythm variability temporary indices (standard deviation NN intervals - SDNN, standard deviation average NN intervals - SDANN- i). In early postoperative period acute reduction of HRV was revealed in patients with severe complications, such as myocarditis, valvular abscesses, severe heart failure associated with cardiac valve destruction as well as the perioperation myocardial infarction. In 3 and 6 months after the IE surgical treatment the number of patients with significant HRV reduction decreased. The significant hypersympaticotonia maintained in patients with previous MI and myocarditis that led to the development of heart failure with the reduction of global left ventricle (LV) contractility. The evidence of significant hypersympaticotonia in patients with type II diabetes mellitus was subject to diabetic neuropathy progressing underlying secondary immune failure in the settings of IE.

Conclusion: The low level of HRV can be used as a complication marker in IE patients including the post-operative period.

Keywords: Infectious endocarditis, heart rate variability, myocarditis, heart failure. 


\section{Introduction}

Heart rhythm variability analysis (HRV) is one of the leading evaluation methods of heart vegetative regulation condition as it reflects the extent of regulatory system strain in response to any stressful effect and cardiovascular system adaptation capacities.

There are results of selected data that investigated clinical value of HRV in arterial hypertension, in congenital heart failure, chronic mitral regurgitation, myocardial infarction. Data are absent on HRV clinical significance in evaluating complication risks during IE. To study HRV by the temporary test results in IE patients prior to the surgery, 6 months after heart valve abnormality surgical correction and to compare that to the progression of complications during the underlying disease.

\section{Materials and methods}

35 IE patients submitted to surgical treatment have been studied; they were on a hospital stay in cardiosurgical departments of GU RSPC “Cardiology” from January 1, 2009 till March 1, 2010.

The study involved IE patients in active disease phase. IE diagnosis was estimated according to standard criteria [Duke,1994] in the presence of positive blood culture and (or) echocardiographic approvals of heart valve lesions. The study includes patients without hemodynamically significant coronary artery lesions according to the data of coronary angiographies performed at the age of 19 to 70 (mean-age $43,6 \pm 2,4$ ). The subjects examined were $27(73,3 \%)$ males and 8 $(26,7 \%)$ females. The primary IE was diagnosed in 22 $(62,9 \%)$ patients, secondary - in $13(37,1 \%)$ from 35 patients who were included in the study. Aortal valve damage (AVD) in IE was revealed in $11(31,4 \%)$ from 35 patients, mitral valve (MV) - in $11(31,4 \%)$ from 35 subjects, simultaneous mitral and aortal valve damage was revealed in $9(25,7 \%)$, tricuspid valve (TV) - in 4 $(11,5 \%)$ patients.

Chronic heart failure symptoms (CHF) of different severity were noted in $100 \%$ of cases. Symptom onset time was different. However, in $18(60 \%)$ of 30 patients IV FC CHF was detected (FC) by New York Heart Association Classification (NYHA), in $8(26,7 \%)$ patients - III FC and in $4(13,3 \%)$ patients' CHF clinical manifestations corresponded to II FC.
Complications during the IE in pre- and post-operational periods, myocarditis, perioperational MI were revealed in $11(31,4 \%)$ out of 35 patients. Intraoperational pyaemic cardiac loci were revealed in $7(20 \%)$ out of 35 subjects: they had their aortic root and/or valve leaflet abscesses. One patient was CT-diagnosed with abscess forming pneumonia. 1 patient of 35 died of heart failure in pre-operation period.

34 patients underwent valve replacement surgery. Six ones $(17,6 \%)$ were implanted with biological prostheses (SJM mitral and tricuspid positions, Sorin tricuspidal position), $28(82,4 \%)$ - mechanical were implanted in mitral and or aortic positions (Planix - E MDM, Planix - T MDM, Planix - T). Moreover, 14 of 34 patients $(41,2 \%)$ received bivalvular correction- aortic valves (AV) and/or MV prosthesis, tricuspid valve plasty using Plankor rings. 10 ones $(29,4 \%)$ underwent monovalvular correction in mitral or aortal positions. One patient $(2,9 \%)$ had MV anterior leaflet quadriangular resection with the advancement onto the leasion region anterior leaflet and MV plasty and hard Plankor ring implantation.

Patient state evaluation who were included into the study was implemented initially (prior to the surgery) - test 1 , on $11,0 \pm 1,0$ day after the valvular correction surgery - test 2, within 3 months after the surgery - test 3 , in 6 months after the surgery - test 4 . HRV was reckoned based on the results of daily ECG Holter monitoring (ECGHM). ECGHM was done using Medilog FD 5 cardiomonitor system by Oxford Instruments Medical. Results of temporary test based on were studied. It was based on statistic programs for reckoning of pronounced quantity of RR interval rates with further interpretation of data obtained.

HRV data interpretation was done according to ESC and North American Society of Pacing and Electrophysiology Working Group (1996). Temporary HRVtest results were evaluated (Time Domain) recommended for usage:

- $\quad$ SDNN (ms) - standard deviation of all RR intervals analyzed (for total HRV assessment).

- SDANN - I (ms) - standard deviation of 5-minute averaged RR interval values which the observation period is divided by (for evaluating of low-frequency variability component). 
- $\quad$ rMSSD (MS) - square root of difference sum of subsequent RR intervals (for evaluating of low-frequency variability component).

Indicators selected were offered by the ESC and North American Society of Pacing and Electrophysiology Working Group for universalization of physiological and clinical studies. The following parameters of temporary test were taken for norm:: SDNN $-141 \pm 39$ ms, SDANN - I $-127 \pm 25 \mathrm{~ms}$, rMSSD $27 \pm 12 \mathrm{~ms}$. The fall-off criterion of 24-hour HRV was con-sidered to be a SDNN value of $<50 \mathrm{~ms}$. A moderate drop criterion of 24-hour HRV was SDNN value of $<100 \mathrm{~ms}$. Two patient groups were singled out by us on every step of examination: group I having a drop of SDNN $<100 \mathrm{~ms}$, group II with fall-off of SDNN $<50 \mathrm{~ms}$.

All reckons were done using personal computer with Excel 7.0 electronic charts («Microsoft», USA). Data obtained have been presented as $\mathrm{M} \pm \mathrm{m}$, where $\mathrm{M}$ - arithmetic mean, $\mathrm{m}$ - standard error of arithmetic mean. Differences between the groups were considered as statistically evident when $\mathrm{p}<0,05$.

\section{Results}

Prior to the surgery, the HRV temporary test assessment was done in 25 patients. HRV was reduced in most cases - in $14(56 \%)$ patients out of 25 examined subjects. Group I included 10 (72\%) patients, Group II $-4(28 \%)$ patients.

Group I demonstrated the reduction in mean indicators reflecting sympathetic activity: SDNN down to $77 \pm 4,5 \mathrm{~ms}, \mathrm{SDANN}-\mathrm{i}$ to $62,6 \pm 4,3 \mathrm{~ms}$. The indicator of rMSSD that reflects parasympathetic activity fluctuated between normal values and made up on average $39,0 \pm 7,3 \mathrm{~ms}$ (the increase of this indicator was noted in $4(40 \%)$ of patients having atrial fibrillation and/or premature beats). 4 patients having severe drop in HRV (Group II) had their temporary test rates evidently different form the mean of Group I and made up: SDNN $-35,56 \pm 4,0 \mathrm{~ms}(\mathrm{p}<0,001)$, SDANN-i- 32,3 $\pm 4,5 \mathrm{~ms}$ $(\mathrm{p}<0,001)$ and $\mathrm{rMSSD} 17,2 \pm 0,5 \mathrm{~ms}(\mathrm{p}<0,001)$.

Mean rates were beyond lower rates of this group of so called "parting points" which is associated with poor prognosis and high death risk. Two of four patients died who had a significant drop in HRV. One patient died of heart failure progression before the operation.
The second one (female) died on a first day after the surgery had been performed. The cause of death was gastrointestinal bleeding that had developed during the preoperational DIC syndrome. One female patient had sepsis with the developing DIC syndrome in preoperational period, myocarditis. One more IE patient had aortic root abscess, myocarditis complication as well as severe aortal insufficiency in preoperational period. Group I did not show any of these complications.

Data of the second examination demonstrated that the situation (compared to the first ex-amination) has not changed substantially. Heart rhythm variability reducing tendencies maintained in $15(57 \%)$ patients of 26 (test $1-56 \%, \mathrm{p}>0,05$ ). The average reduction in SDNN (Group I) was revealed in $67 \%$ of patients (test $1-72 \%, p>0,05$ ). Severe drop (Group II) was revealed in $5(33 \%)$ patients (test $128 \%, \mathrm{p}>0,05)$.

SDNN and SDANN- I indicators were evidently different in Group I and II and made up: SDNN 75,9 $\pm 3,0$ $\mathrm{ms}$ and $45,0 \pm 2,1 \mathrm{~ms}(\mathrm{p}<0,05)$ accordingly, SDANNi $64,1 \pm 4,6 \mathrm{~ms}$ and 42,5 $\pm 2,4 \mathrm{~ms}(\mathrm{p}<0,001)$, accordingly. rMSSD value was $28,7 \pm 6,2 \mathrm{~ms}$ and $16,8 \pm 1,1$ $\mathrm{ms}(\mathrm{p}>0,05)$, accordingly in Groups I and II and it flits between the normal values. Retrospective analysis showed that in the second examination in group 2 that had a moderated decrease of HRV there were no significant complications in the course of in-patient period.

Among those having a significant drop in HRV - one out of $5(20 \%)$, demonstrated a persistent sepsis with progressive DIC syndrome in early post-operational period ( 2 weeks after the surgery), heart failure accompanied by developing postoperational myocarditis. He died 3 months later after the surgical valve correction. Two ones $(40 \%)$ developed perioperational postoperational myocardial infarction (MI). Maintenance of postoperational hypersymathicotonia can be perhaps explained by preserved and/or progressive heart failure and postoperation MI development.

Past MI is inevitably accompanied by left ventricular (LV) remodeling. The latter is able to cause LV dysfunction. It is considered that changes in heart geometry may cause sympathetic fiber afferent impulsation acceleration due to mechanical extension of receptors. This sympathetic activation weakens vagal effects on heart. There data present on the relations of daily SDNN drop and LV dysfunction. It is worth pointing 
that any acute MI (including the perioperational) is itself accompanied by hypersympathicotonia. According to one of the hypotheses, during the HRV drop, after the past MI, cardio-cardinalsympatho-sympathic and sympatho-vagal reflexes get involved. So, detection of pronounced hypersympathicotonia in IE patients can be one of criteria of poor prognosis in the progression of complications in pre- and postoperational periods.

In 3 months after the operation the heart rhythm variability is noted in 9 (45\%) of 20 patients examined (tests 1 and $256 \%$ and $57 \%$, accordingly, $\mathrm{p}>0,05)$. In the third checkup a severe drop was noted in 2 put of 9 (22\%) patients - Group II, moderate in 7 (78\%) - Group I.

SDNN, SDANN- i indices were evidently different in groups I and II (pronounced according to the qualitative values of dividing values of SDNN) and made up correspondingly: SDNN 70,2 $\pm 6,7 \mathrm{~ms}$ and 43,9 $\pm 3,0 \mathrm{~ms}$ $(\mathrm{p}<0,01)$, SDANN- i $-61,5 \pm 5,9 \mathrm{~ms}$ and 40,7 $\pm 1,6 \mathrm{~ms}$ $(p<0,01)$. Mean values rMSSD reflecting parasymapthic activity were significantly lower in group II than in group I and made up 39,8 $\pm 13,0 \mathrm{~ms}$ and 9,5 $\pm 0,7 \mathrm{~ms}$, $(\mathrm{p}<0,05)$, accordingly. In group I the rMSSD value was above $39 \mathrm{~ms}$ in $2(22,2 \%)$ subjects who were registered with atrial fibrillation.

One female patient having a severe drop of HRV registered during the third checkup developed postoperation myocardial infarction that contributed to heart failure with LV global contractility reduction (EF - 22/24 $\%)$. Another patient having a severe drop of HRV was diagnosed with myocarditis in preoperational period.

Six months later (the fourth checkup) 5 of 14 (35\%) subjects having their HRV parameters evaluated maintained temporary BCP test indices drop: HRV drop incidence was lower than in tests 1 and 2, 56\% and 57\% accordingly, $\mathrm{p}<0,05)$. A moderate reduction in $\mathrm{HRV}$ was registered in $4(80 \%)$ of 5 patients, severe - in one $(20 \%)$. Mean-values of HRV in group I were the following: SDNN $-79,5 \pm 6,2 \mathrm{~ms}$, SDANN- i $-70,8 \pm 6,1$ ms, rMSSD 25,3 $\pm 4,4 \mathrm{~ms}$. A patient with a severe drop of HRV turned out to have diabetes mellitus, and such a notable HRV drop that emerged in 6 months after the surgery could have been an outcome of progressive diabetic neuropathy accompanied by a secondary immune insufficiency.

In general it might be said that 3 and 6 months later after the operation the hypersympath-icotonia frequency had reduced according to the HRV data. A huge drop in SDNN, SDANN- i values maintained in subjects having severe heart failure or a concomitant diabetes mellitus.

\section{Conclusion}

1. A sudden drop in HRV was noted in subjects having severe complications, as my-ocarditis, valvular abscesses, severe heart failure associated with heart valvular apparatus destruction and perioperational myocardial infarction.

2. Three and six months after the operative treatment of IE the number of patients having a considerable HRV rate drop decreased. A pronounced hypersympathicotonia was preserved in patients who underwent myocardial infarction and myocarditis which contributed to , heart failure with global LV contractility reduction. The appearance of severe hypersympathicotonia in patient with II type diabetes mellitus was subject to diabetic neuropathy progression accompanied by a secondary IE immune insufficiency.

3. Low HRV may be used as a marker of complications in IE subjects including the post-operation period.

Disclosure and conflicts of interest:

Conflicts of interest were not reported.

Corresponding author:

Dr. Natalia Feaktsistava

n0902@yandex.ru 